$\begin{array}{cc}\text { Programa de Pós-Graduação em Engenharia de Produção - PPGEP } & \\ \text { Laboratório de Qualidade de Vida - LaQVida } & \text { REVISTA BRASILEIRA DE } \\ \text { Universidade Tecnológica Federal do Paraná - UTFPR } & \text { Ponta Grossa - PR - Brasil } \\ \text { v. 06, n. 04, out./dez. 2014, p. } 241-249 & \text { QUALIDADE DE VIDA }\end{array}$

\title{
Qualidade de vida: avaliação de dependentes químicos em processo de reabilitação
}

\section{Quality of life: assessment of drug addicts in the rehabilitation process}

\author{
José Carlos da Silva Lopes \\ Universidade Federal de Alagoas - UFAL - Maceió - Alagoas - Brasil \\ jcarlosjrcd@yahoo.com.br \\ Mayara Nakíria Tavares da Rocha \\ Universidade Federal de Alagoas - UFAL - Maceió - Alagoas - Brasil \\ may_nakiria@hotmail.com \\ David dos Santos Calheiros \\ Universidade Federal de São Carlos - UFSCar - São Carlos - São Paulo - Brasil \\ davidcalheiros@hotmail.com \\ Tereza Angélica Lopes de Assis \\ Universidade Federal de Alagoas - UFAL - Maceió - Alagoas - Brasil \\ terezaangelica@gmail.com
}

\section{RESUMO}

OBJETIVO: Investigar a qualidade de vida de dependentes químicos em processo de reabilitação. MÉTODOS: Realizou-se um estudo quantitativo descritivo, de corte transversal, com uma população de dependentes químicos. Participaram da pesquisa 50 homens. Utilizou-se como critério para comprovar a relação de dependência química a Classificação Estatística Internacional de Doenças e Problemas Relacionados à Saúde CID-10. Os dados referentes à qualidade de vida (QV) foram coletados a partir da aplicação do Questionário WHOQOL-Bref, os dados sociodemográficos foram alcançados por meio de um roteiro de entrevista semidirigida e os dados relativos aos aspectos socioeconômicos emergiram com base no Critério de Classificação Econômica Brasil. Para avaliar esses dados foi utilizada a análise estatística descritiva. Para realizar a análise estatística, as informações coletadas foram transferidas a um banco de dados previamente criado e, a seguir, realizou-se uma avaliação percentual e estatística para apurar os resultados. A etapa seguinte foi a análise descritiva, com a apresentação das medidas de tendência central e dispersão para as variáveis contínuas e número e percentual das variáveis categoriais.

RESULTADOS: A média de idade da amostra foi de 29,6 anos $(\mathrm{dp}=9,0)$ pertencente, em sua maioria, à classe socioeconômica baixa (Classes C, D e E). Na avaliação da QV o domínio Psicológico apresentou o maior escore médio, seguido pelos domínios Físico, Relações Sociais e Meio Ambiente. Observou-se que os toxicômanos da classe social baixa apresentaram menores escores de QV, tendo sido o domínio Psicológico mais afetado.

CONCLUSÕES: Os drogaditos apresentaram padrões de QV geral positiva, porém, quando comparasse o aspecto socioeconômico verificou-se que a classe baixa está relacionada a menores valores indicativos de QV.

PALAVRAS-CHAVE: Qualidade de Vida. Dependentes Químicos. Reabilitação. 


\section{ABSTRACT}

OBJECTIVE: To investigate the quality of life for drug addicts in the rehabilitation process. METHODS: We conducted a descriptive quantitative study, cross-sectional, with a population of drug addicts. A total of 50 men participated in the survey. The International Statistical Classification of Diseases and Related Health Problems ICD -10 were used as a criterion to prove the relationship of chemical dependency. The data relating to quality of life were collected from the application of the WHOQOL-Bref questionnaire, the sociodemographic data were obtained through a semistructured interview script and data on socioeconomic aspects emerged based on the Brazil Economic Classification Criteria. To analyze these data was employed descriptive statistical analysis. To perform the statistical analysis, the data were transferred to a database previously created, and then carried out a percentage evaluation and statistical to determine the results. The next step was a descriptive analysis, with the presentation of measures of central tendency and dispersion for continuous variables and number and percentage for categorical variables.

RESULTS: The average age of the sample was 29.6 years $(\mathrm{SD}=9.0)$ belonging mostly to the low socioeconomic class (Classes C, D and E). In evaluating the quality of life the psychological domain had the highest mean score, followed by Physical, Social Relations and Environment domain domains. It was observed that the drug addicts of low social class had lower QOL scores, having been the most affected psychological domain.

CONCLUSIONS: We conclude that drug addicts had generally positive standards of quality of life, but when we compare the socioeconomic aspect it was found that the lower class is related to lower values indicative of QOL.

KEYWORDS: Quality of life. Drug Addicts. Rehabilitation.

\section{Introdução}

Nas últimas décadas, muito se tem discutido sobre drogas e dependência química, cuja definição refere-se a um padrão de uso intenso que envolve descontrole sobre a quantidade ou a frequência com que se consome, acompanhado de desejo forte, muitas vezes incontrolável, de utilizar a droga (TAUB; ANDREOLI, 2004).

Nessa perspectiva, a droga torna-se a questão central da vida do dependente químico, passando a estabelecer um duo indissociável, indivíduo-droga, em que tudo o que não é pertinente a essa relação passa a constituir pano de fundo na existência do dependente. E esta situação permanece indissociável enquanto a droga for capaz de propiciar alteração da percepção de uma realidade vista como insuportável, respondendo assim pela manutenção do equilíbrio do indivíduo (MACCOUN, 2004).

Esse fenômeno da dependência química ou toxicomania cresceu assustadoramente, e tornou foco da atenção mundial, dada à dimensão que adquiriu o seu impacto na sociedade moderna (MORAIS, 2010; PULCHERIO et al., 2010). Estima-se que 200 milhões de pessoas no mundo consumam alguma droga ilícita e 2,1 bilhões drogas lícitas, como cigarro e álcool (MORAIS, 2010). Esse consumo vem se difundindo, cada vez mais, entre pessoas mais jovens (CARLINI et al., 2006; MOUTINHO; LOPES, 2008; FORMIGA et al., 2009; PULCHERIO et al., 2010; BENINCASA, 2010). E, só no Brasil, a cada ano, o uso indevido de drogas custa 7,9\% do Produto Interno Bruto (PIB), que se perde nos prejuízos provenientes da queda na produtividade e gastos com tratamentos de saúde (MOUTINHO; LOPES, 2008).

A dependência de drogas, notadamente, provoca danos à saúde do indivíduo. O abuso ou uso nocivo sempre está associado a algum tipo de prejuízo, quer em termos biológicos, psicológicos ou sociais (LIMA, 2008). Tais prejuízos podem ter caráter: agudo (durante a intoxicação ou overdose) ou crônico (produzindo alterações mais duradouras e, até, irreversíveis) (MARQUES; CRUZ, 2000). Drogas como álcool, tabaco, maconha, cocaína, crack, entre outras, destroem o caráter, 
comprometem o sentido realístico, diminuem ou acabam com o senso de responsabilidade, fragilizam a família e o próprio indivíduo, que soma para si uma série de alterações físicas, químicas e emocionais (COLODEL et al., 2009).

Os danos provocados pela dependência de drogas alteram vários aspectos da vida do indivíduo e, paulatinamente, provocam uma redução da qualidade de vida (QV) (VENTEGODT; MERRICK, 2003; LAUDET; MORGEN; WHITE, 2006; BENINCASA, 2010; BREKKE et al., 2010), cuja definição é dada como o nível de satisfação com a vida, a autoestima, a percepção de bem-estar psicológico, as condições de trabalho e o bem-estar geral (NAHAS, 2001; KLUTHCOVSKY, 2005; BITTAR, 2009).

Avaliação da QV na perspectiva do uso, abuso e dependência química, torna-se algo muito importante, tendo em vista que a política do Ministério da Saúde para a atenção integral aos usuários de álcool e outras drogas (BRASIL, 2004) descreve que há uma maior vulnerabilidade para o uso indevido dessas substâncias, naqueles indivíduos que estão insatisfeitos com a sua QV, que possuem uma precária condição de saúde, que não detêm informações minimamente adequadas sobre a questão de álcool e drogas ou que ainda apresentem uma integração comunitária deficiente.

Todavia, devido à escassez de estudos que façam essa conexão entre o uso de drogas e a QV, ainda é difícil ter conhecimento da dimensão dessa relação (LAUDET; MORGEN; WHITE, 2006), sendo necessário o desenvolvimento de novas pesquisas nesse âmbito. Nesse cenário, objetivou-se nessa pesquisa investigar a QV de dependentes químicos em processo de reabilitação.

\section{Metodologia}

O estudo apresenta um caráter transversal, exploratório e quantitativo, sendo realizado na Casa do Servo Sofredor, pertencente à instituição Juvenopólis em Maceió - AL, caracterizada como uma casa de acolhimento para dependentes químicos em processo de reabilitação, que aguardam admissão nas comunidades terapêuticas.

A pesquisa propôs-se a estudar a QV de homens acolhidos na instituição, na faixa etária de 18 a 60 anos, que mantinham com o álcool e/ou outras drogas uma relação diagnosticável de dependência, segundo diretrizes propostas pela Classificação Estatística Internacional de Doenças e Problemas Relacionados à Saúde - CID -10 (OMS, 1993), no período de janeiro a abril de 2011. No primeiro contato com os prováveis sujeitos da pesquisa, foi esclarecida a sua importância e os seus objetivos, desta feita, os que aceitaram participar, assinaram o Termo de Consentimento Livre e Esclarecido (TCLE).

A amostra forma da pesquisa contou com 50 homens, para os quais foram aplicados os seguintes instrumentos:

a) Entrevista clínica semidirigida e estruturada com o propósito de coletar informações demográficas (idade, profissão, pessoas com as quais reside, entre outras) e referentes ao uso de drogas, abordando aspectos como: primeiro contato e as substâncias já utilizadas; as repercussões desse uso para suas vidas; internações anteriores; experiências em família com uso de drogas, entre outros aspectos;

b) Critério de Classificação Econômica Brasil - Elaborado pela Associação Nacional de Empresas de Pesquisa (ANEP, 2000) o qual tem por objetivo estimar o poder de compra das pessoas e famílias urbanas, baseando-se em pontos calculados a partir da posse de itens, considerando assim as seguintes classes socioeconômicas A1, A2, B1, B2, C, D e $\mathrm{E}$;

c) WHOQOL-Bref - instrumento que avalia a QV, sendo uma versão abreviada do WHOQOL-100. É composto por quatro domínios: Físico, Psicológico, Relações sociais e Meio ambiente, contendo 26 questões, sendo 2 questões gerais de QV e as demais 24 representam cada uma das 24 facetas que compõe o instrumento original (FLECK et al., 2000). O instrumento fornece um perfil da QV obtido através dos escores dos quatro domínios, onde quanto mais alto os escores, melhor é a QV, indicada através da percepção individual. As duas questões gerais, que o compõe, são examinadas 
separadamente: onde a primeira questão revela a percepção do indivíduo sobre a sua QV e a segunda questão revela a percepção do indivíduo sobre a sua saúde. As demais 24 questões possuem uma pontuação que varia de 1 a 5 . Desta forma para o cálculo manual de cada domínio há uma equação que resulta em um escore bruto. $\mathrm{O}$ escore bruto é então convertido em um escore transformado, utilizando-se uma tabela de referência. $\mathrm{O}$ método de transformação converte os escores brutos em uma escala de 4 a 20, comparável com o WHOQOL-100, e em uma escala de 0 a 100 (THE GROUP WHOQOL, 1994). Os escores dos domínios podem ser agrupados e então revelar um escore único para a QV.

Para realizar a análise estatística, as informações coletadas foram transferidas a um banco de dados previamente criado e, a seguir, realizou-se uma avaliação percentual e estatística para apurar os resultados. A etapa seguinte foi a análise descritiva, com a apresentação das medidas de tendência central e dispersão para as variáveis contínuas e número e percentual das variáveis categoriais. O Programa Epi Info versão 3.5.3 foi utilizado para análise dos dados.

Quanto aos aspectos éticos esta pesquisa foi conduzida com autorização formal dos setores envolvidos, tendo os preceitos éticos vigentes, estabelecidos pela Resolução 466/2012 do Conselho Nacional de Saúde (BRASIL, 2012), obedecidos e a identidade dos pacientes e profissionais envolvidos preservada. A pesquisa foi previamente avaliada e aprovada pelo Comitê de Ética em Pesquisa da Universidade Federal de Alagoas (CEP/UFAL), sob o número 020619/2010-61.

\section{Resultados}

A amostra do estudo foi constituída por 50 homens, dependentes químicos, que demandaram o serviço em uma casa de acolhimento do município de Maceió, no período de janeiro a abril/ 2011. Nesse contexto, a Tabela 1 foi elaborada, a fim de discorrer sobre os aspectos sociodemográficos que os envolviam:

Tabela 1 - Características sociodemográficas dos dependentes químicos atendidos na Casa do Servo Sofredor de Maceió

\begin{tabular}{lcc}
\hline VARIÁ VEIS & $\mathbf{N}^{\mathbf{0}}$ & $\mathbf{\%}$ \\
\hline Faixa Etária & & \\
\hline $18-30$ & 29 & 58 \\
$30-45$ & 16 & 32 \\
$45-60$ & 5 & 10 \\
\hline Escolaridade & & \\
\hline Analfabetos & 5 & 10 \\
Alfabetizados & 7 & 14 \\
Fundamental ou mais & 38 & 76 \\
\hline Ocupação & & \\
\hline Serviços gerais & 39 & 78 \\
Autônoma & 9 & 18 \\
Técnica & 1 & 2 \\
Profissão graduada & 1 & 2 \\
\hline Renda Familiar & & \\
\hline$<1$ salário mínimo & 18 & 36 \\
1 salário mínimo & 17 & 34 \\
2 ou mais salários mínimos & 15 & 30 \\
\hline Classe Social A e B & & \\
\hline Sim (alta e média) & 15 & 30 \\
Não (baixa) & 35 & 70 \\
\hline Moradia & & \\
\hline Abrigo & 1 & 2 \\
Família 1 (pais e irmãos) & 27 & 54 \\
Família 2 (cônjuge e fillhos) & 8 & 16 \\
Parentes & 2 & 4 \\
Rua & 4 & 8 \\
Sozinho & 8 & 16 \\
\hline \multicolumn{1}{c}{ Fonte: Autoria prória (2011). } \\
\hline
\end{tabular}


Os resultados da avaliação da QV dos sujeitos da pesquisa estão alocados na Tabela 2, onde são representados os escores médios na escala de 0 a 100 e os desvios-padrão obtidos para os domínios do WHOQOL:

Tabela 2 - Escores médios e desvios-padrão para os domínios do WHOQOL-Bref

\begin{tabular}{lcccc}
\hline Domínio & N & Média & Mediana & Desvio-padrão \\
\hline Psicológico & 50 & 65,9 & 66,6 & 18,5 \\
Físico & 50 & 63,6 & 64,3 & 19,3 \\
Relações sociais & 50 & 62,9 & 66,6 & 23,9 \\
Meio ambiente & 50 & 54,6 & 53,1 & 17,0 \\
\hline \multicolumn{4}{c}{ Fonte: Autoria prória (2011). }
\end{tabular}

Observa-se que, entre os domínios, o Psicológico apresentou o maior escore médio ( $\mathrm{dp}=$ 18,5), seguido pelos domínios Físico, Relações Sociais e, conferindo a última posição ao domínio do Meio ambiente, com o menor escore médio $(\mathrm{dp}=17,0)$.

Quando tais domínios de QV aferidos pelo WHOQOL-Bref foram comparados com a classificação socioeconômica da população estudada, encontrou-se que em todas as áreas os toxicômanos não pertencentes à classe social $\mathrm{A}$ e $\mathrm{B}$ apresentaram menores escores de $\mathrm{QV}$, tendo sido o domínio Psicológico mais afetado, passando do valor médio 70,56 $(\mathrm{dp}=13,69)$ para 63,94 $(d p=20,09)$. Os escores dos diferentes domínios estão descritos na Tabela 3.

Tabela 3 - Relação dos escores de QV para os domínios do WHOQOL-Bref com a classe socioeconômica

\begin{tabular}{|c|c|c|c|c|c|c|c|}
\hline \multirow{3}{*}{ Domínio } & \multicolumn{7}{|c|}{ Classe Social A e B } \\
\hline & \multicolumn{3}{|c|}{ Sim } & \multicolumn{4}{|c|}{ Não } \\
\hline & $\mathbf{N}$ & Média & Desvio- Padrão & $\mathbf{N}$ & Média & Desvio-Padrão & $\mathbf{P}$ \\
\hline Psicológico & 15 & 70,56 & 13,69 & 35 & 63,94 & 20,09 & 0,323 \\
\hline Físico & 15 & 64,76 & 16,47 & 35 & 63,17 & 20,68 & 0,866 \\
\hline Relações Sociais & 15 & 64,44 & 18,76 & 35 & 62,38 & 26,07 & 0,982 \\
\hline Meio Ambiente & 15 & 57,92 & 14,89 & 35 & 53,31 & 17,90 & 0,373 \\
\hline
\end{tabular}

Fonte: Autoria prória (2011).

\section{Discussão}

De acordo com o que fora apresentado, observou-se que na população estudada a média de idade foi de 29,6 anos ( $\mathrm{dp}=9,0$ ), com um mínimo de 18 e um máximo de 53 anos, tendo o grupo etário de 18 a 30 anos incompletos como o mais frequente, fato coincidente com o que foi descrito em outros estudos envolvendo a dependência química (CARLINI et al., 2006; FORMIGA et al., 2009; LIMA, 2008). Assim, de modo geral, pôde-se encontrar a dependência química afetando um perfil composto por homens jovens, solteiros, de baixa escolaridade, sem vínculo empregatício e de classe social baixa.

$\mathrm{Na}$ avaliação da QV dos dependentes químicos através do WHOQOL-Bref, o Domínio Ambiental obteve o menor escore médio entre os demais domínios. Tendo em vista que esse domínio pode ser compreendido por meio de aspectos envolvendo o ambiente no lar, recursos financeiros, cuidados de saúde e sociais - disponibilidade e qualidade, oportunidades de adquirir novas informações e habilidades, participação e oportunidades de recreação/lazer, ambiente físico (poluição/ruído/trânsito/clima) e transporte (THE WHOQOL GROUP, 1994), fato que se correlacionado ao perfil da amostra estudada, bem como, aos problemas de saúde associados ao uso de drogas.

Ao comparar a QV com a classificação socioeconômica, as análises descritivas dos dados coletados permitiram expor valores médios de QV com a interferência da variável socioeconômica. Diante disso, constatou-se que para todos os domínios, as médias dos escores de QV foram menores nos toxicômanos não pertencentes à classe social $\mathrm{A}$ e $\mathrm{B}$, ou seja, os pertencentes ao baixo nível socioeconômico. 
Nessa perspectiva, sabe-se que a pobreza é potencialmente atuante no fenômeno da toxicomania, onde a população de baixa renda utiliza a droga, em algumas situações, para amenizar angustias e sofrimentos inerentes à fome e a miséria (LEANDRINI, 2004). Desta forma, o consumo de substâncias psicoativas comporta-se como uma espécie de anulação/anestesia do mal-estar social e das subjetividades (CONTE, 2008). O indivíduo que vive em condições precárias estará em contato com restrições (falta de perspectiva profissional, dificuldade de relacionamento humano decorrente da situação social, problemas de saúde, dificuldades de acesso à informação e formação cultural), situação que repercute em sua vida emocional, gerando sentimentos de angústia, nervosismo, desânimo e tristeza que poderão ser pseudominorados através do uso de drogas psicotrópicas (LEANDRINI, 2004).

No entanto, a pobreza não é o único fator a agir e influenciar no fenômeno da toxicomania. O uso de substâncias psicoativas, comumente, envolve uma complexa combinação de fatores genéticos, psicológicos, familiares, socioeconômicos e culturais (BRASIL, 2004; BENINCASA, 2010; FORMIGA et al., 2009; LAUDET; MORGEN; WHITE, 2006). Desta forma, o uso, o abuso e a dependência são fenômenos muito complexos que não podem ser reduzidos a uma dimensão exclusiva, e que diversas variáveis estão relacionadas com um maior ou menor uso, bem como, com um maior ou menor índice de QV (BENINCASA, 2010).

A relação entre o uso de drogas e a $\mathrm{QV}$ costuma ser inversa, obedecendo à premissa de que quanto mais se consome drogas, menor é a QV (BENINCASA, 2010). Essa concepção também foi descrita em outros estudos, a exemplo de Fassino et al. (2004), que investigando QV e transtornos de personalidade em italianos usuários de heroína, percebeu que ela foi inferior quando comparada aos não usuários. Ou ainda, Lima (2002) que avaliando os domínios do WHOQOL-Bref em alcoolatras de acordo com o grau de dependencia, encontrou escores menores de QV para os alcoolatras de estado grave.

No entanto, não se deve ignorar o fato de que o consumo de drogas, em princípio, tem intensidade cujo prejuízo não é visível, proporcionando prazer, alegria, euforia, relaxamento, bemestar, ou seja, contribuindo para um autorelato de boa QV (BENINCASA, 2010; GALDUROZ et al., 2010; SCHENKER; MINAYO, 2004). É seu gradativo caminhar para o abuso e dependência química, que se associa à paulatina redução da QV (BENINCASA, 2010; BREKKE et al., 2010; LAUDET; MORGEN; WHITE, 2006; VENTEGODT; MERRICK, 2003).

Entre os domínios do WHOQOL-Bref utilizados na avaliação da QV e comparados à variável socioeconômica, o domínio Psicológico foi o mais afetado, quando comparados aos demais. Esse fato merece destaque, pois ao avaliar QV e esperança em toxicodependentes, Morais (2010) afirma que o domínio Psicológico é o único a explicar significativamente as variâncias nos índices de esperança e, desse modo, quando afetado remete a um baixo nível de esperança, correlacionado com sentimento de desvalorização, solidão e finalização da vida. Em contrapartida, um elevado senso de segurança, controle e estabilidade são muito necessários e conferem força e resistência ao uso de substâncias numa recuperação (LAUDET; MORGEN; WHITE, 2006).

Portanto, embora não sejam recentes, os estudos de QV ainda carecem de interesses voltados para o grupo do usuário de droga dependente (LAUDET; MORGEN; WHITE, 2006; MORAES, 2010;). Na literatura, notou-se que existem poucos trabalhos que abordam o grupo neste quesito, ao passo que é largamente estudado os tipos de drogas consumidas de acordo com o aspecto sociodemográfico.

\section{$5 \quad$ Conclusão}

Em relação à QV dos drogaditos acolhidos, conclui-se que os escores apresentados pelos quatro domínios do WHOQOL-Bref revelaram valores sugestivos de positividade para a QV do grupo estudado. A QV dos drogaditos não pertencentes às classes socioeconômicas $\mathrm{A}$ e $\mathrm{B}$ foi reduzida em todos os domínios quando comparada ao grupo pertencente às classes $\mathrm{A}$ e $\mathrm{B}$, sendo mais evidente no domínio Psicológico. Entretanto, o aumento da QV não foi associado significativamente ao aspecto socioeconômico. Ainda são necessários estudos detalhados nesta área, 
proporcionando um maior entendimento da relação entre $\mathrm{QV}$, uso de drogas e classes socioeconômicas.

\section{Referências}

ANEP. Associação Nacional de Empresas de Pesquisas. Dados com base no levantamento sócio econômico. São Paulo: IBOPE, 2000.

BENINCASA, M. Avaliação da qualidade de vida e uso de drogas em adolescentes do município de São Paulo. 2010. 353f. Tese (Doutorado em Psicologia da Aprendizagem, do Desenvolvimento Humano e da Personalidade) - Instituto de Psicologia da Universidade de São Paulo, São Paulo, 2010.

BITTAR, A. M. Qualidade de vida de cuidadores de idosos. 2009. 99 f. Dissertação (Mestrado em Saúde e Gestão do Trabalho) - Centro de Ciências da Saúde, Universidade do Vale do Itajaí, Itajaí, 2009.

BRASIL. A política do Ministério da Saúde para a atenção integral a usuários de álcool e outras drogas. 2. ed. Brasília, DF: Ministério da Saúde, 2004.

BRASIL. Resolução n 466, de 12 de Dezembro de 2012. Brasília, DF: Conselho Nacional de Saúde, 2012. Disponível em: <http://conselho.saude.gov.br/resolucoes/2012/Reso466.pdf>. Acesso em: 23 jun. 2014.

BREKKE, M.; VETLESEN, A.; HOIBY, L.; SKEIE, I. Quality of life among patients in drugassisted rehabilitation programmes. Tidsskr Nor Laegeforen, v. 130, n. 13, p. 1340-1342, 2010. crossef

CARLINI, E. A.; GALDURÓZ, J. C. F; NOTO, A. R.; FONSECA, A. M.; CARLINI, C. M.; OLIVEIRA, L. G.; NAPPO, A. S.; MOURA, Y. G.; SANCHEZ, Z. VAN DER. M. II

Levantamento domiciliar sobre o uso de drogas psicotrópicas no Brasil: estudo envolvendo as 108 maiores cidades do país - 2005. São Paulo: CEBRID - Centro Brasileiro de Informação sobre Drogas Psicotrópicas: UNIFESP - Universidade Federal de São Paulo, 2006.

COLODEL, E. V.; SILVA, E. L. F. M.; ZIELAK, J. C.; ZAITTER, W.; MICHEL-CROSATO, E.; PIZZATTO, E. Alterações bucais presentes em dependentes químicos. Revista Sul-Brasileira de Odontologia, v. 6, n. 1, p. 44-48, 2009.

CONTE, M. Desafios na juventude: drogas, consumismo e violências. Educação e Realidade, Porto Alegre, v. 33, n. 2, p.131-146, jul./dez. 2008.

FASSINO, S.; DAGA, G. A.; DELSEDIME, N.; ROGNA, L.; BOGGIO, S. Quality of life and personality disorders in heroin abusers. Drug and Alcohol Dependence, v. 76, n. 1, p. 73-80, 2004. crossef

FLECK, M. P. A.; LOUZADA, S.; XAVIER, M.; CHACHAMOVICH, E.; VIEIRA, G.; SANTOS, L.; PINZON, V. Aplicação da versão em português do instrumento abreviado de avaliação da qualidade de vida “WHOQOL-Bref”. Revista de Saúde Pública, São Paulo, v. 34, n. 2, p. 178-183, 2000. rossef 
FORMIGA, L. T.; SANTOS, R. C. S.; DUMCKE, T. S.; ARAUJO, R. B. Comparação do perfil de dependentes químicos internados em uma unidade de dependência química de Porto Alegre/RS em 2002 e 2006. Clinical and Biomedical Research, Porto Alegre, v. 29, n.2, p. 120-126, 2009.

GALDUROZ, J. C. F.; SANCHES, Z. M.; OPAYELE, E. S.; NOTO, A. R.; FONSECA, A. M.; GOMES, P. C. S.; CARLINI, E. A. Fatores associados ao uso pesado de álcool entre estudantes das capitais brasileiras. Revista Saúde Pública, São Paulo, v. 44, n. 2, p. 267-273, 2010. Crossef

KLUTHCOVSKY, A. C. G. C. Qualidade de vida dos agentes comunitários de saúde de um município do interior do Paraná. 2005. 118 f. Tese (Mestrado em Enfermagem) - Escola de Enfermagem de Ribeirão Preto, Universidade de São Paulo, Ribeirão Preto, 2005.

LAUDET, A. B.; MORGEN, K.; WHITE, W. L. The role of social supports, spirituality, religiousness, life meaning and affiliation with 12-step fellowships in quality of life satisfaction among individuals in recovery from alcohol and drug problems alcoholism treatment quarterly. Alcoholism Treatment Quarterly, v. 24, n. 1-2. p. 33-73, 2006. rossef

LEANDRINI, T. M. M. Avaliação do impacto da implantação do Centro de Atenção Psicossocial de São Carlos na população de usuários de drogas em tratamento. 2004. 63f. Trabalho de Conclusão de Curso (Graduação em Psicologia) - Centro de Educação e Ciências Humanas, Universidade Federal de São Carlos, São Paulo, 2004.

LIMA, A. F. B. S. Qualidade de vida em pacientes do sexo masculino dependentes de álcool. 2002. 90f. Dissertação (Mestrado em Medicina) - Universidade Federal do Rio Grande do Sul, Porto Alegre, 2002.

LIMA, A. P. Características do sono e qualidade de vida em dependentes de cocaína. 2008. $90 f$. Dissertação (Mestrado em Neurologia) - Departamento de Neurologia da Faculdade de Medicina, Universidade de São Paulo, São Paulo, 2008.

MACCOUN, R. Alcohol \& drug abuse: population thinking as an adjunct to the clinical trial perspective. Psychiatric Services, v. 55, n, 5, p. 509-515, 2004. crossef

MARQUES, A. C. P. R.; CRUZ, M. S. O adolescente e o uso de drogas. Revista Brasileira de Psiquiatria, São Paulo, v. 22, n. 2, p. 32-36, 2000. Crossef

MORAIS, P. O. I. Avaliação da qualidade de vida e esperança em toxicodependentes infectados e não infectados com VIH. 2010. 115f. Dissertação (Mestrado em Psicologia Clinica e da Saúde) - Faculdade de Ciências Humanas e Sociais, Universidade Fernando Pessoas, Porto, 2010.

MOUTINHO, E. C. V. S; LOPES, G. T. Enfermeiro do Programa Saúde da Família: conceitos e crenças sobre drogas e modelos teóricos explicativos. Revista de Enfermagem da UERJ, Rio de Janeiro, v. 16, n. 1, p. 51-57, 2008.

NAHAS, M. V. Atividade física, saúde e qualidade de vida: conceitos e sugestões para um estilo de vida ativo. Londrina: Midiograf, 2001

ORGANIZAÇÃO MUNDIAL DA SAÚDE. Classificação internacional de doenças e problemas relacionados à saúde. 10. rev. São Paulo: Centro Colaborador da OMS para a Classificação de Doenças em Português, 1993. 
PULCHERIO, G.; STOLF, A. S.; PETTENON, M.; FENSTERSEIFER, D. P.; KESSLER, F. Crack: da pedra ao tratamento. Revista da Associação Médica do Rio Grande do Sul, Porto Alegre, v. 54, n. 3, p. 337-343, jul./set. 2010.

SCHENKER, M.; MINAYO, M. C. S. A importância da família no tratamento do uso abusivo de drogas: uma revisão da literatura. Cadernos de Saúde Pública, Rio de Janeiro, v. 20, n. 3, p. 649659, maio/jun. 2004.

TAUB, A.; ANDREOLI, P. B. A. Guia para a família: cuidando da pessoa com problemas relacionados com álcool e outras drogas. São Paulo: Atheneu; 2004.

THE WHOQOL GROUP. The development of the World Health Organization quality of life assessment instrument (the WHOQOL). In: ORLEY, J.; KUYKEN, W. (Org.) Quality of life assessment: international perspectives. Heidelberg: Springer Verlag, 1994.

VENTEGODT, S.; MERRICK, J. Psychoactive Drugs and Quality of Live. Scientific World Journal, v. 18, n. 3, p. 694-706, 2003. crossef 